\title{
Recapitulating in vivo-like plasticity of glioma cell invasion along blood vessels and in astrocyte-rich stroma
}

\author{
Pavlo Gritsenko $^{1}$ - William Leenders ${ }^{2}$ Peter Friedl ${ }^{1,3,4}$
}

Accepted: 3 August 2017 / Published online: 19 August 2017

(C) The Author(s) 2017. This article is an open access publication

\begin{abstract}
Diffuse invasion of glioma cells into the brain parenchyma leads to nonresectable brain tumors and poor prognosis of glioma disease. In vivo, glioma cells can adopt a range of invasion strategies and routes, by moving as single cells, collective strands and multicellular networks along perivascular, perineuronal and interstitial guidance cues. Current in vitro assays to probe glioma cell invasion, however, are limited in recapitulating the modes and adaptability of glioma invasion observed in brain parenchyma, including collective behaviours. To mimic in vivo-like glioma cell invasion in vitro, we here applied three tissue-inspired 3D environments combining multicellular glioma spheroids and reconstituted microanatomic features of vascular and interstitial brain structures. Radial migration from multicellular glioma spheroids of human cell lines and patient-derived xenograft cells was monitored using (1) reconstituted basement membrane/hyaluronan interfaces representing the space along brain vessels; (2) 3D scaffolds generated by multi-layered mouse astrocytes to reflect brain interstitium; and (3) freshly isolated mouse brain slice culture ex vivo.
\end{abstract}

Peter Friedl

peter.friedl@radboudumc.nl; pfriedl@mdanderson.org

1 Microscopical Imaging of the Cell, Department of Cell Biology, Radboud Institute for Molecular Life Sciences, Radboud University Medical Center, Nijmegen, The Netherlands

2 Department of Pathology, Radboud University Medical Center, Nijmegen, The Netherlands

3 Department of Genitourinary Medical Oncology, David H Koch Center for Applied Research of Genitourinary Cancers, The University of Texas, MD Anderson Cancer Center, Houston, TX, USA

4 Cancer Genomics Centre (CGC.nl), 3584 Utrecht, The Netherlands
The invasion patterns in vitro were validated using histological analysis of brain sections from glioblastoma patients and glioma xenografts infiltrating the mouse brain. Each 3D assay recapitulated distinct aspects of major glioma invasion patterns identified in mouse xenografts and patient brain samples, including individually migrating cells, collective strands extending along blood vessels, and multicellular networks of interconnected glioma cells infiltrating the neuropil. In conjunction, these organotypic assays enable a range of invasion modes used by glioma cells and will be applicable for mechanistic analysis and targeting of glioma cell dissemination.

Keywords Glioma invasion - Organotypic culture · Perivascular invasion · Astrocyte scaffolds · Multicellular networks

\section{Introduction}

Gliomas represent the most common primary brain tumor type in adults, with glioblastoma as one of the most detrimental cancers in humans (Wen and Reardon 2016). The high lethality of glioma patients is mainly caused by diffuse invasion of glioma cells into the brain parenchyma, the extent of which typically precludes curative surgical resection and radiotherapy (Cuddapah et al. 2014). This diffuse invasive character is a relatively unique characteristic of gliomas and rarely seen in other brain cancers (Lenting et al. 2017). The structures of the brain tissue along which glioma cells migrate are complex and their microanatomic and molecular organization varies. Guiding structures include myelinated axons and astrocyte processes (white matter tracks) as well as basement membranes of blood vessels and the meninges (perivascular 
tracks) (Gritsenko et al. 2012; Cuddapah et al. 2014). Both brain regions contain hyaluronan as main component of the brain extracellular matrix (ECM) (Zimmermann and Dours-Zimmermann 2008). Disseminating glioma cells orient preferentially along aligned myelinated fibers and astrocyte processes throughout the white matter (Gritsenko et al. 2012; Cuddapah et al. 2014). In parallel, glioma cell invasion occurs along the interface between blood vessels and brain parenchyma (Farin et al. 2006; Gritsenko et al. 2012; Cuddapah et al. 2014). Glioma cells invade the brain tissue either individually, after detaching from neighboring glioma cells, or as cohesive groups preferentially moving along blood vessels (Farin et al. 2006; Winkler et al. 2009; Assanah et al. 2009; Hirata et al. 2012; Watkins et al. 2014; Baker et al. 2014; Krusche et al. 2016). In addition, glioma cells may form multicellular networks with long filaments connecting glioma cells in vivo, and these networks were recently implicated in glioma cell invasion and resistance signaling (Osswald et al. 2015).

In recent years, different assays were developed to model glioma microenvironments of the brain tissue and test the extent and mechanisms of glioma cell invasion (Rao et al. 2014; Rape et al. 2014). Widely used 2D assays are based on coating of the culture dish with ECM molecules, including laminin, fibronectin or collagen (Nakada et al. 2013; Chen and Nalbantoglu 2014). However, these models lack crucial parameters of 3D brain environments which modulate migration mechanisms, including (1) low substrate stiffness, (2) anatomically complex 3D organization, (3) 3D space confinements which enable adhesion-dependent and adhesion-independent migration, and (4) molecularly rich ECM composition maintained by brain cells and containing chemotactic factors (Rape et al. 2014). Reconstituted 3D migration assays are based on natural or synthetic hydrogels with various molecular components, including fibrillar collagen, reconstituted basement membrane (rBM) rich in laminin and type IV collagen, and composite hydrogels containing polyacrylamide, fibronectin and/or cross-linked hyaluronan (Gordon et al. 2003; Ulrich et al. 2009; Yang et al. 2010; Ananthanarayanan et al. 2011). In unperturbed brain, fibrillar collagens are expressed mainly along blood vessels but not in the parenchyma (Bellail et al. 2004; Gritsenko et al. 2012), and upregulated in a subset of clinical gliomas in the tumor mass and perivascular regions (Huijbers et al. 2010; Payne and Huang 2013; Motegi et al. 2014). Type I collagen scaffolds are effectively invaded by glioma cells (Kaufman et al. 2005; Yang et al. 2010; Frolov et al. 2016); however, the relevance of collagen as substrate for diffuse glioma cell infiltration beyond the tumor core remains unclear (Rape et al. 2014). rBM and cross-linked hyaluronan both represent key components of the brain stroma and, like synthetic hydrogels, provide soft environments similar to brain tissue; however, they lack other adhesion ligands and cell-derived brain structures, such as astrocyte networks and myelinated axons (Gritsenko et al. 2012).

Migration assays comprising brain-derived cells in monolayer culture, including primary astrocytes, provide 2D interaction scaffolds for glioma cells (Oliveira et al. 2005; Rath et al. 2013; Hong et al. 2015). Astrocyte monolayers support glioma cell invasiveness via gap-junction communications and by secretion of promigratory molecules (Oliveira et al. 2005; Rath et al. 2013; Hong et al. 2015). As 3D modification, primary rat astrocytes were combined with electrospun nanofiber scaffolds, and this approach revealed a contribution of astrocytes to single cell migration of glioma cells by secreting migration-enhancing factors (Rao et al. 2014). However, both astrocyte monolayers and electrospun scaffolds lack the complexity and, likely, softness of 3D brain stroma (Rape et al. 2014).

Consequently, live brain slice assays are considered as "gold standard" recapitulating the complexity of brain tissue; however, they support mainly perivascular but not parenchymatous routes of glioma cell invasion (Alfi et al. 2002; Miao et al. 2015; Fayzullin et al. 2016). To this end, we hypothesize that the complexity of glioma invasion requires the combined application of a set of complementary in vitro models to enable an adaptive range of glioma invasion types, including collective perivascular invasion and network-like interstitial invasion patterns (Osswald et al. 2015, 2016).

We here developed a set of 3D assays mimicking brainlike structures to analyse glioma cell invasion patterns from 3D multicellular spheroids. When compared to in vivo invasion of the same cell types in the mouse brain and to histopathology of human lesions, each assay delivers dedicated in vivo-like invasion programs, including single cell migration, collective sheets and strands, and/or multicellular glioma networks.

\section{Materials and methods}

\section{Antibodies and reagents}

The following antibodies were used: anti-mouse $\beta$-catenin (mouse clone 14/beta-catenin, 1:100, BD Biosciences); anti-human $\beta$-catenin (rabbit polyclonal, 1:1000, Abcam); anti-mouse N-cadherin (mouse, clone GC-4, 1:200, Sigma); anti-mouse laminin (rabbit polyclonal, 1:100, Sigma); antihuman collagen-IV (mouse, clone Col-94, 1:300, Sigma); anti-human vimentin (rabbit, SP20 clone, human specific, 1:300, Thermo Scientific), anti-bovine glial fibrillary acidic protein (GFAP) (chicken polyclonal, 1:1000, Abcam); antihuman nestin (rabbit polyclonal, human specific, 1:100, Millipore). Primary antibodies were visualized with secondary AlexaFluor-conjugated goat-anti-mouse, goat-anti-rabbit or goat-anti-chicken polyclonal antibodies (Invitrogen; $5 \mu \mathrm{g}$ / 
$\mathrm{ml})$. For background controls, isotype-matched unspecific mouse IgG (BD Biosciences) was used for monoclonal antibody stainings, and for polyclonal antibody stainings samples were incubated only with secondary AlexaFluorconjugated antibodies. Cell nuclei were stained with 4',6-diamidino-2-phenylindole (DAPI; $2.5 \mu \mathrm{g} / \mathrm{ml}$ ). F-actin was labelled with AlexaFluor-conjugated phalloidin (Invitrogen). Growth factor-reduced reconstituted basement membrane (rBM) (Matrigel, BD Biosciences; $9.8 \mathrm{mg} / \mathrm{ml}$ ) was used for rBM interface culture.

\section{Cell lines and culture}

Human glioblastoma E-98 and E-468 cells were maintained as patient-derived xenografts by serial subcutaneous (E-98) and intracerebral (E-468) inoculation without in vitro culture (Claes et al. 2008). E-468 cells were freshly isolated from mouse brain 7-8 days prior to each spheroid preparation for migration assays to minimize adaptation to in vitro culture. E-468 cells were maintained in neurobasal medium (Invitrogen) supplemented with human EGF $(20 \mathrm{ng} / \mathrm{ml})$, human bFGF (20 ng/ml), B27 Supplement (1:50), L-glutamine ( $2 \mathrm{mM}$ ) (all from Invitrogen), heparin $(2 \mu \mathrm{g} / \mathrm{ml}$, Sigma), penicillin $(100 \mathrm{U} / \mathrm{ml})$ and streptomycin $(100 \mu \mathrm{g} / \mathrm{ml}$; both PAA). Cells were maintained for up to two passages (E-468) in 2D culture on flasks coated with growth factor-reduced rBM (BD Biosciences; $30 \mu \mathrm{g} / \mathrm{ml}$ in PBS). Accutase digestion (10 min, 400-600 units/ml; Sigma) was used for cell detachment and dissociation of multicellular spheroids. A subline of E-98 cells was propagated in vitro in flasks for up to passage 35 . Human glioblastoma U-251MG cells (kind gift from Dr. J. Schalkwijk, Nijmegen) were maintained permanently in in vitro culture. Primary mouse astrocytes immortalized with SV40 large T-antigen and additionally transformed with retrovirus pBabe puro H-Ras V12 (kindly provided by Amparo Acker-Palmer, Institute of Cell Biology and Neuroscience and BMLS, Goethe University Frankfurt, Germany) were maintained as described (Sawamiphak et al. 2010; Depner et al. 2016). H2B/eGFP-expressing U-251 and E-98 cells were generated by lentiviral transduction with pLenti6.2/V5-DEST ${ }^{\mathrm{TM}}$ Gateway (Invitrogen) containing histone2B/eGFP. For in vitro invasion assays murine astrocytes and human E-98 and U-251 cells were maintained in Dulbecco's Modified Eagle's Medium (DMEM; Invitrogen) supplemented with $10 \%$ fetal bovine serum (Sigma-Aldrich), penicillin $(100 \mathrm{U} / \mathrm{ml})$ and streptomycin $(100 \mu \mathrm{g} / \mathrm{ml}$; both PAA), L-glutamine (2 $\mathrm{mM}$, Invitrogen) and sodium pyruvate (1 mM, Invitrogen).

\section{Generation of glioma cell spheroids}

Glioma cell spheroids were generated using the hanging drop method (Korff and Augustin 1998). Cells were cultured in DMEM until subconfluency, detached with $1 \mathrm{mM}$ EDTA/0.075\% trypsin or with Accutase (400-600 units/ $\mathrm{ml}$; Sigma), washed with PBS, and maintained for $24 \mathrm{~h}$ in complete DMEM/methylcellulose (2.4\%; Sigma) as hanging droplets $(25 \mu \mathrm{L})$ containing 1000 (U-251, E-468) or 2000 (E-98) cells.

\section{Reconstituted basement membrane interface migration assays}

3D rBM/hyaluronan interface cultures were generated by polymerizing growth factor-reduced $\mathrm{rBM}$ on a culture dish (30 $\mathrm{min}, 37^{\circ} \mathrm{C}$ ), followed by addition of glioma spheroids over polymerized $\mathrm{rBM}$ in complete DMEM with further incubation $\left(2 \mathrm{~h}, 37^{\circ} \mathrm{C}, 10 \% \mathrm{CO}_{2}\right)$, replacement of media by complete DMEM supplemented with sodium hyaluronate (Sigma, Cat: 53747, from Streptococcus equi, $\mathrm{Mr} \sim 1.5-1.8 \times 10^{6} \mathrm{Da}, 10 \mathrm{mg} / \mathrm{ml}$ ) or methylcellulose (Sigma, Cat: M6385, viscosity $25 \mathrm{cP}, 15 \mathrm{mg} / \mathrm{ml}$ ) and incubation for 24-48 h. For rBM-plastic interface cultures, glioma spheroids were placed on 96-well plates (Greiner Bio One, PS, F-bottom, $\mu$ Clear, Black, CELLSTAR) coated with growth factor reduced $\mathrm{rBM}(30 \mu \mathrm{g} / \mathrm{ml}$, diluted in PBS, preadsorbed overnight at $4{ }^{\circ} \mathrm{C}$ ). After spheroid addition, cultures were incubated in complete DMEM or neurobasal media $\left(2 \mathrm{~h}, 37{ }^{\circ} \mathrm{C}, 10 \% \mathrm{CO}_{2}\right)$, overlaid with growth factor reduced $\mathrm{rBM}(5 \mathrm{mg} / \mathrm{ml}$, diluted in PBS) and incubated to allow rBM polymerization $\left(30 \mathrm{~min}, 37^{\circ} \mathrm{C}\right.$ ). After 24 or $48 \mathrm{~h}$ of migration culture in complete DMEM or neurobasal media, samples were fixed (4\% PFA, $30 \mathrm{~min}, \mathrm{RT}$ ) and analysed by bright-field or confocal microscopy.

\section{D astrocyte scaffold invasion assay}

3D astrocyte-derived scaffolds were generated by immortalized murine astrocytes maintained at high cell density in 96-well plates (Greiner Bio One, PS, F-bottom, $\mu$ Clear, Black, CELLSTAR; 20,000 cells/well, coated with growth factor-reduced $\mathrm{rBM}$ ) for 2-3 days resulting in consolidated 3D scaffolds of up to 3-4 cell layers in height. Glioma cell spheroids expressing H2B/eGFP were cultured on top of astrocyte scaffolds (4-5 spheroids/well) for 2 days, fixed (4\% PFA, 30 min, RT), and stained to visualize glioma cells (human specific anti-vimentin antibody), astrocytes and ECM proteins.

\section{Organotypic mouse brain slice invasion assay}

To probe glioma cell invasion into 3D brain slices, the assay from (Montana and Sontheimer 2011) was modified for multicellular spheroid culture. Brains from female mice (5-6 weeks old; Charles River or Jackson Laboratories) were dissected, using the strain C57BL/6-Tg(TcraTcrb)1100Mjb/ 

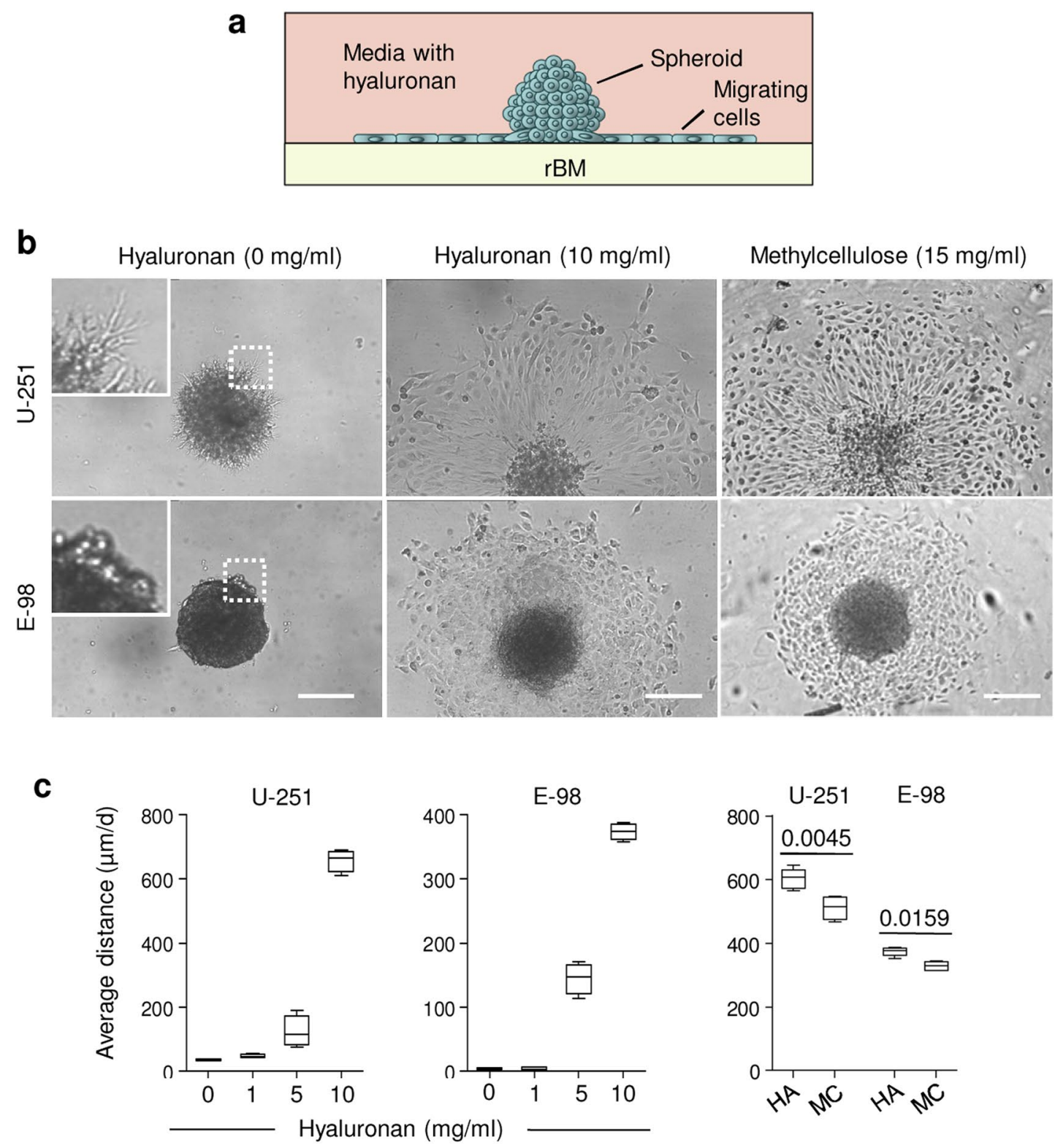

d
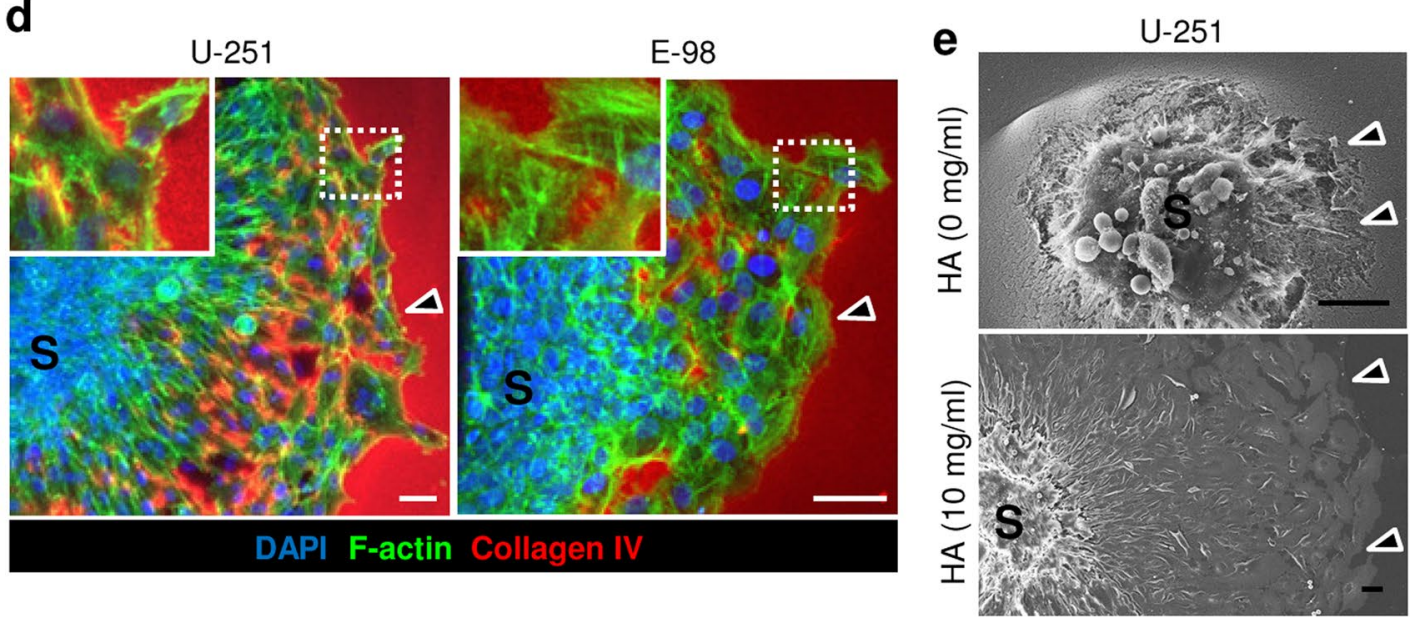
4Fig. 1 Reconstituted basement membrane/hyaluronan interface migration assay. a Assay design. b Radial migration of U-251 and E-98 cells from spheroids along the rBM-hyaluronan (HA) or methylcellulose (MC) interface after 1 day of culture, detected by bright-field microscopy. c Average distance migrated by U-251 and E-98 cells along the $\mathrm{rBM} / \mathrm{HA}$ or $\mathrm{rBM} / \mathrm{MC}$ interface at different concentration of HA or MC; values display medians (black line), 25/75 percentiles (boxes) and maximum/minimum (whiskers) from three independent experiments. $p$ values, Mann-Whitney test. d 3D projection from confocal z-stack of U-251 and E-98 cell migration from multicellular spheroids (S) along rBM/HA interface $(10 \mathrm{mg} / \mathrm{ml} \mathrm{HA}$ concentration). Arrowheads indicate the invasion front. e Scanning electron microscopy of U-251 cells after 1 day of radial migration from spheroids (S) on rBM in media without or with HA $(10 \mathrm{mg} / \mathrm{ml})$. Arrowheads, invasion front with signs of degradation of rBM (HA, $0 \mathrm{mg} / \mathrm{ml}$ ) or without $\mathrm{rBM}$ degradation $(\mathrm{HA}, 10 \mathrm{mg} / \mathrm{ml}$ ). Scale bars $200 \mu \mathrm{m}(\mathbf{b}), 50 \mu \mathrm{m}(\mathbf{d}, \mathbf{e})$

Crl (OT1) which was crossed with B6.Cg-Tg(CAGDsRed*MST)1Nagy/J (dsRed) in our laboratory. Brains were freshly sectioned as $400 \mu \mathrm{m}$-thick tissue slices using a vibratome (Leica, VT1000 s). Slices were maintained on transwell insert membranes (Costar, 12-well plate; $8 \mu \mathrm{m}$ pore diameter) in complete DMEM $\left(37{ }^{\circ} \mathrm{C}, 5 \% \mathrm{CO}_{2}\right)$ for $1 \mathrm{~h}$. Glioma cell spheroids expressing $\mathrm{H} 2 \mathrm{~B} / \mathrm{eGFP}$ were added on top of brain slices (8-10 spheroids per slice), cultured in complete DMEM for $48 \mathrm{~h}$, fixed (4\% PFA, $1 \mathrm{~h}, 20^{\circ} \mathrm{C}$ ), washed and stained with human-specific antivimentin antibody to discriminate glioma cells from DsRedexpressing blood vessels.

\section{Confocal microscopy and quantification of glioma cell migration}

Confocal microscopy (Olympus FV1000) was performed using long working distance $20 \times$ NA 0.50 and $40 \times$ NA 0.80 objectives at a vertical step size of $2-3 \mu \mathrm{m}$. 3D reconstruction of Z-stacks was performed using Imaris V.6.1.5 (Bitplane).

For quantitative image analysis, operator-assisted image segmentation of bright-field or confocal 3D stacks was performed [Fiji software, V.1.49 g (Schindelin et al. 2012)]. The average cell migration distance representing radial migration of glioma cells from spheroids was calculated according to the following formula:

Average distance of cell migration

$$
=\sqrt{\text { Total cell area } / \pi}-\sqrt{\text { Spheroid area } / \pi} \text {. }
$$

\section{Glioblastoma xenografts in mouse brain}

Female athymic Balb/C nu/nu mice (6-8 weeks old) were obtained from Charles River Laboratories and maintained under specific pathogen-free conditions at the central animal facility of Radboudumc, Nijmegen. The animal experiments were approved by the Ethical Committee on
Animal Experiments of the Radboud University, Nijmegen, The Netherlands (RU-DEC-2013-251) and performed in accordance with the Dutch Animal Experimentation Act and the European FELASA protocol (www.felasa.eu/guidelines. php). U-251-Fluc-mCherry and E-98-Fluc-mCherry parental cells (Wurdinger et al. 2008; Mir et al. 2010) were cultured as spheroids in neurobasal media for 1 month, enzymatically dissociated by Accutase digestion, and intracranially implanted $\left(5 \times 10^{5}\right.$ cells in $20 \mu \mathrm{L}$ PBS $)$ by guided injection into the right parieto-occipital hemisphere of isofluraneanesthetized mice $2 \mathrm{~mm}$ from the midline.

\section{$3 D$ reconstruction of glioma lesions}

Paraffinized clinical samples from four anonymized glioma patients (primary glioblastoma) were obtained from the archives of the Department of Pathology, Radboudumc, Nijmegen. Informed patient consent and ethical committee approval for the use of (archival) brain tissue was obtained and the material was used in a manner compliant with the Declaration of Helsinki. Slices of $100 \mu \mathrm{m}$ thickness were obtained by microtome slicing (HM 340E, Thermo Scientific Microm), deparaffinized (100\% xylene), gradually rehydrated (sequential 100, 96, 70, 50\% v/v ethanol/water), heated for antigen retrieval $\left(98{ }^{\circ} \mathrm{C}, 15 \mathrm{~min}\right.$ in Tris-EDTA, $\mathrm{pH} 9.0)$, incubated with blocking solution (0.1\% Tween-20 and $1 \%$ bovine serum albumin in PBS) and stained with antibodies. To reach saturated antibody conditions and efficient washing in the 3D sample, prolonged incubation periods with primary and secondary antibodies and each washing step (0.1\% tween-20/PBS; $0.05 \% \mathrm{NaN} 3)$ were $8-24 \mathrm{~h}$ at room temperature to ascertain antigen saturation and complete removal of unbound antibody. The presence of nestin in the absence of astrocytic and neuronal markers GFAP and myelin basic protein (MBP), respectively, was used to identify glioma cells (Kitai et al. 2010). $200 \mu \mathrm{m}$ thick sections from glioblastoma xenografts (U-251, E-98 and E-468 cells) in mouse brains were obtained after fixation (4\% PFA, $20 \mathrm{~h}$ ) by vibratome slicing (Leica, VT1000 s) followed by counterstaining to detect murine astrocytes by anti-GFAP pAbs, basement membranes by anti-laminin pAb and glioma cells by human-specific anti-vimentin or anti-nestin pAbs.

\section{Results and discussion}

\section{Glioma cell migration along reconstituted basement membrane interfaces}

To recapitulate perivascular glioma cell invasion along interfaces formed by brain parenchyma and basement membranes, we used polymerized rBM, which comprises structural glycoproteins constituting basement membrane (Albini 


\section{a}

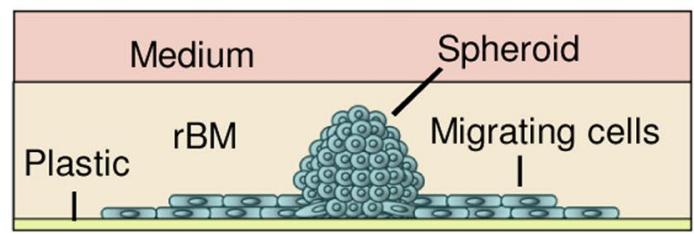

b

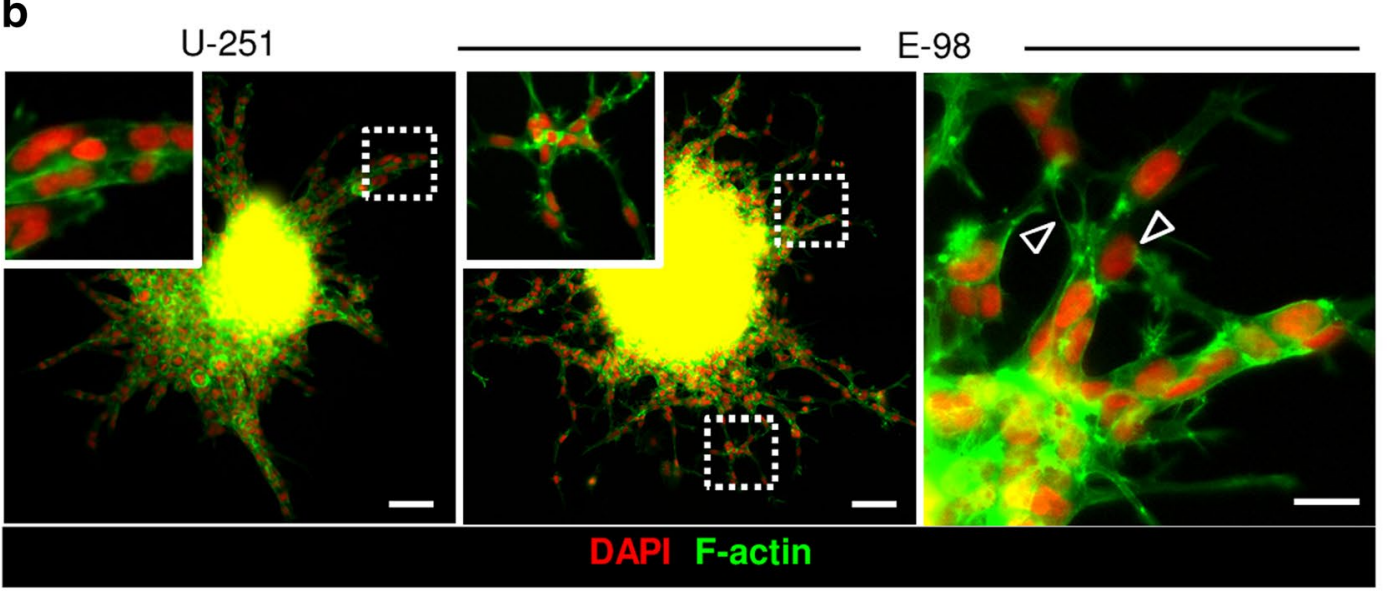

C

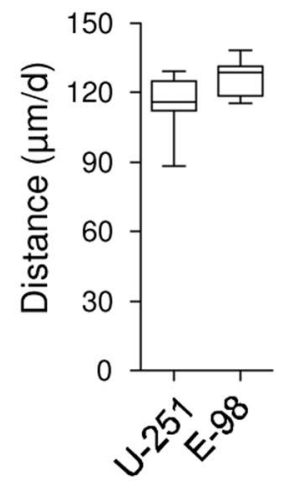

d

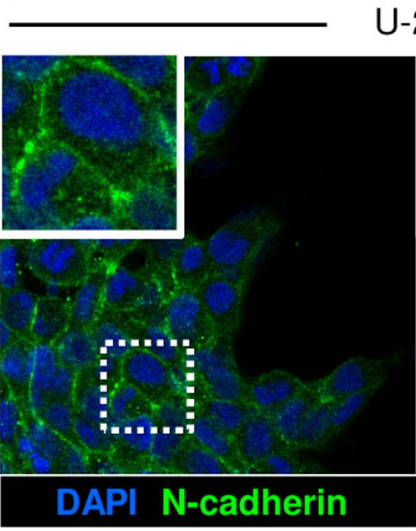

U-251
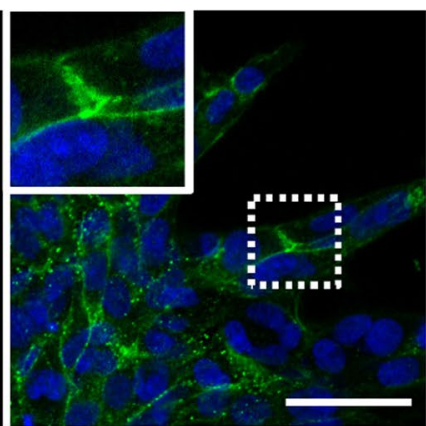

DAPI $\beta$-catenin

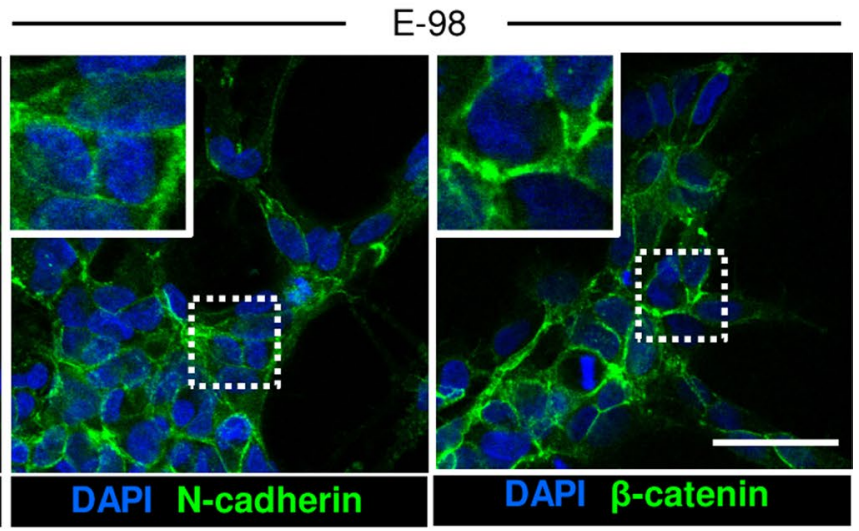

e

$\mathrm{U}-251$

\section{E-98}

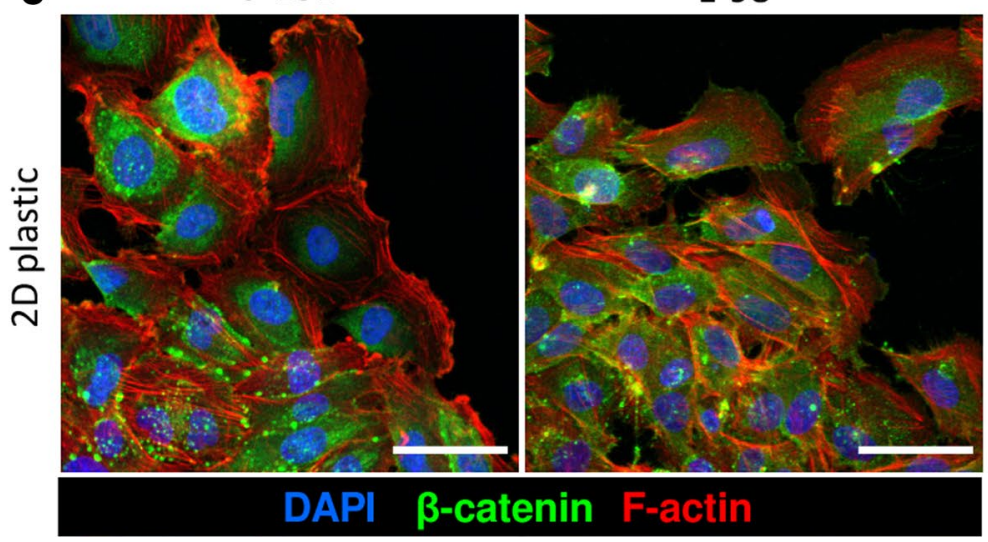

et al. 1987; Hughes et al. 2010), overlaid with hyaluronan, the most abundant component of interstitial brain ECM (Zimmermann and Dours-Zimmermann 2008) (Fig. 1a).
When overlaid on 3D rBM without hyaluronan in the supernatant, U-251 glioma cells invaded into, but not along the rBM, and E-98 cells failed to establish radial migration but 
4Fig. 2 rBM-plastic interface migration assay. a Assay design. b Overviews of U-251 and E-98 cells after 2 days of radial migration from spheroids under rBM in neurobasal media. Arrowheads, focal cell-cell interactions. c Average migration distance of U-251 and E-98 cells under rBM. Values display median (black line), 25/75 percentiles (boxes) and maximum/minimum (whiskers) from three independent experiments. d Molecular topology of adherens junction proteins in U-251 and E-98 cells migrating under rBM. Images were obtained by epifluorescence (b) and confocal microscopy (d). e Maximum z-projection of U-251 and E-98 cells after 2 days of emigration from multicellular spheroids maintained on polystyrene surface coated with rBM. Scale bars $100 \mu \mathrm{m}$ (b), $20 \mu \mathrm{m}$ (zoomed insert b), $50 \mu \mathrm{m}(\mathbf{d}, \mathbf{e})$ grew as compact spheroids (Fig. 1b). When hyaluronan was overlaid, both U-251 and E-98 cells developed sheet-like migration (Fig. 1b) with the speed increasing in dependence of the hyaluronan concentration reaching up to $10 \mathrm{mg} /$ $\mathrm{ml}$, a supra-physiological concentration at which hyaluronan formed a viscous, semi-solid solution (Fig. 1c, d). In gliomas, extracellular hyaluronan concentrations may range from 0.2 up to $5 \mathrm{mg} / \mathrm{ml}$ (Delpech et al. 1993; Sykova and Nicholson 2008). In control experiments using methylcellulose as non-physiological, inert polysaccharide in combination with rBM, migration of U-251 and E-98 cells was a

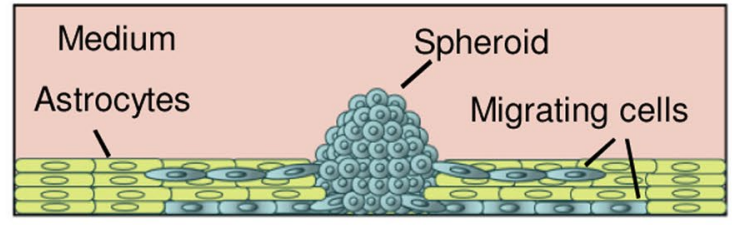

b
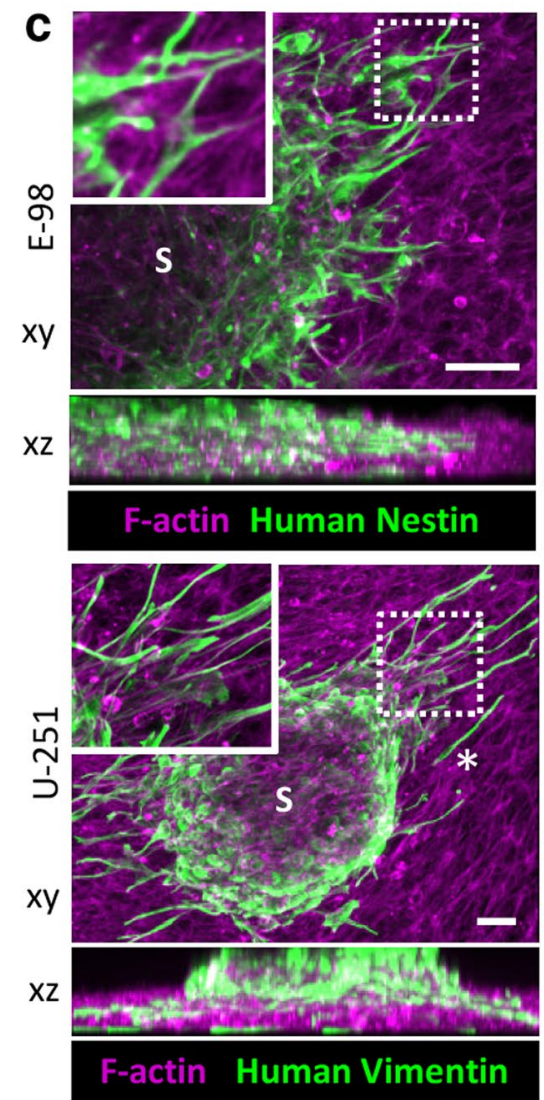
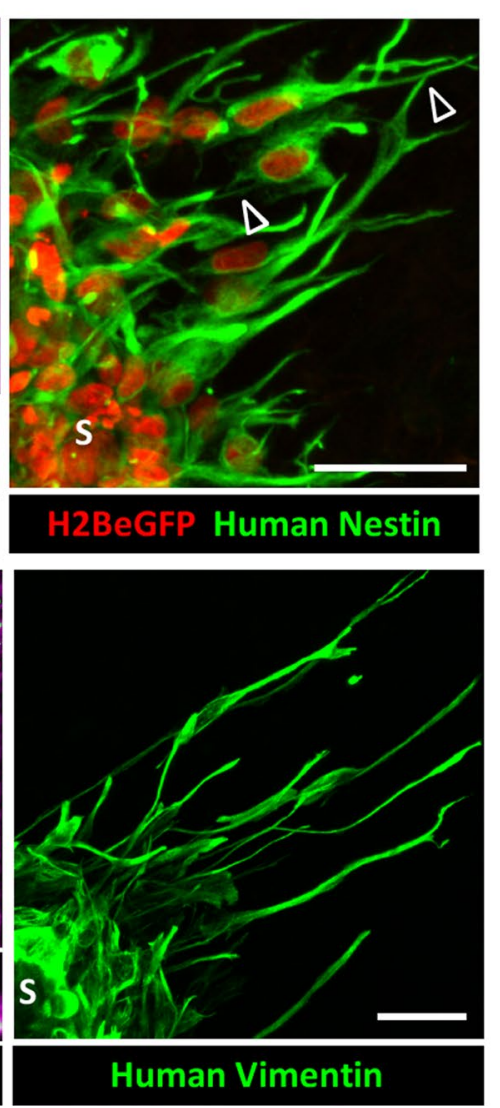
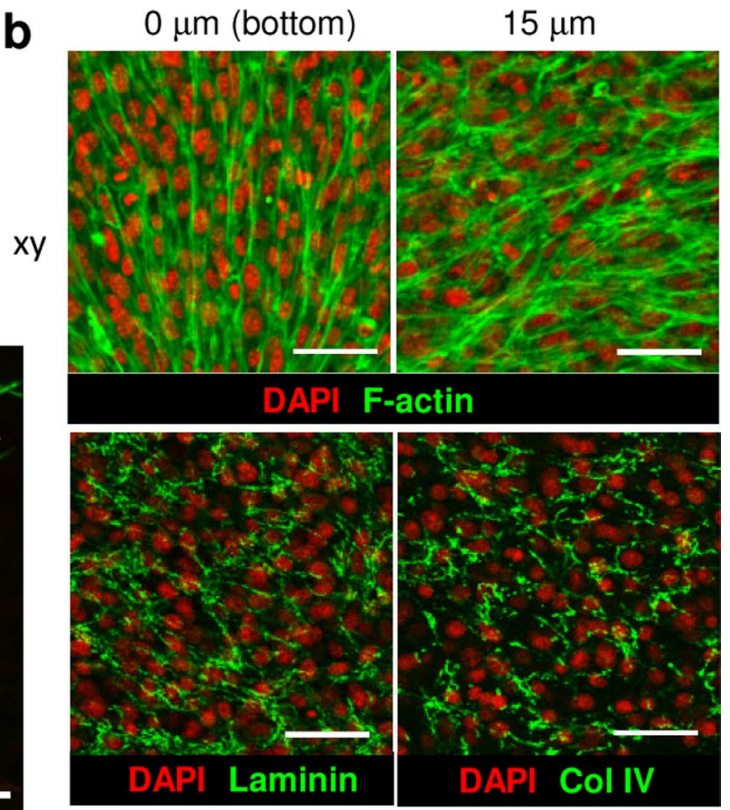

d

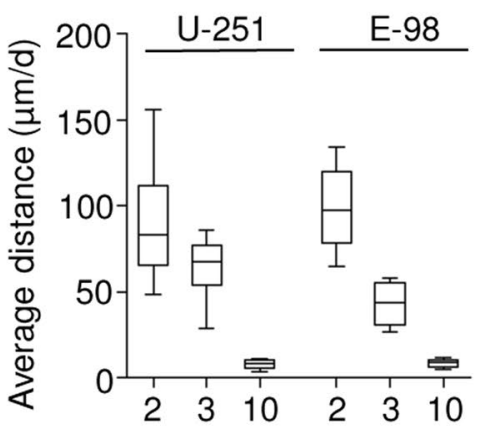

Age of astrocyte culture (d)
Fig. 3 3D astrocyte scaffold invasion assay. a Assay design. b Confocal xy-sections of astrocyte culture (3 days) stained for F-actin, laminin and collagen type IV (Col IV). c 3D reconstruction (confocal Z-stack, $90 \mu \mathrm{m}$, horizontal and orthogonal projections) of E-98 and U-251 cell invasion from spheroids (S) into 3-day old mouse astrocyte scaffolds. Glioma cells were identified by vimentin staining with human-specific antibody and constitutive expression of H2BeGFP in the nucleus, and murine astrocytes using phalloidin (F-actin). Arrowheads point to contacts between glioma cells via dendrite-like filaments. Asterisk, detached single cell. d Average migration distance of U-251 and E-98 cells invading astrocyte scaffolds matured for 2, 3 or 10 days before addition of glioma spheroids. Values display median (black line), 25/75 percentiles (boxes) and maximum/minimum (whiskers) from three independent experiments. Scale bars $50 \mu \mathrm{m}$ 
a

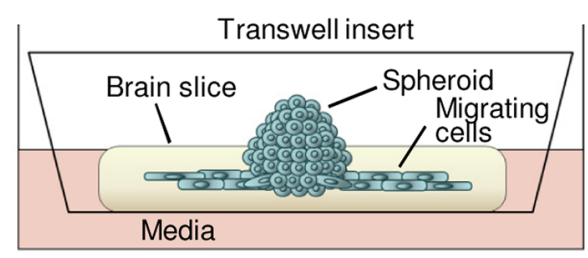

b
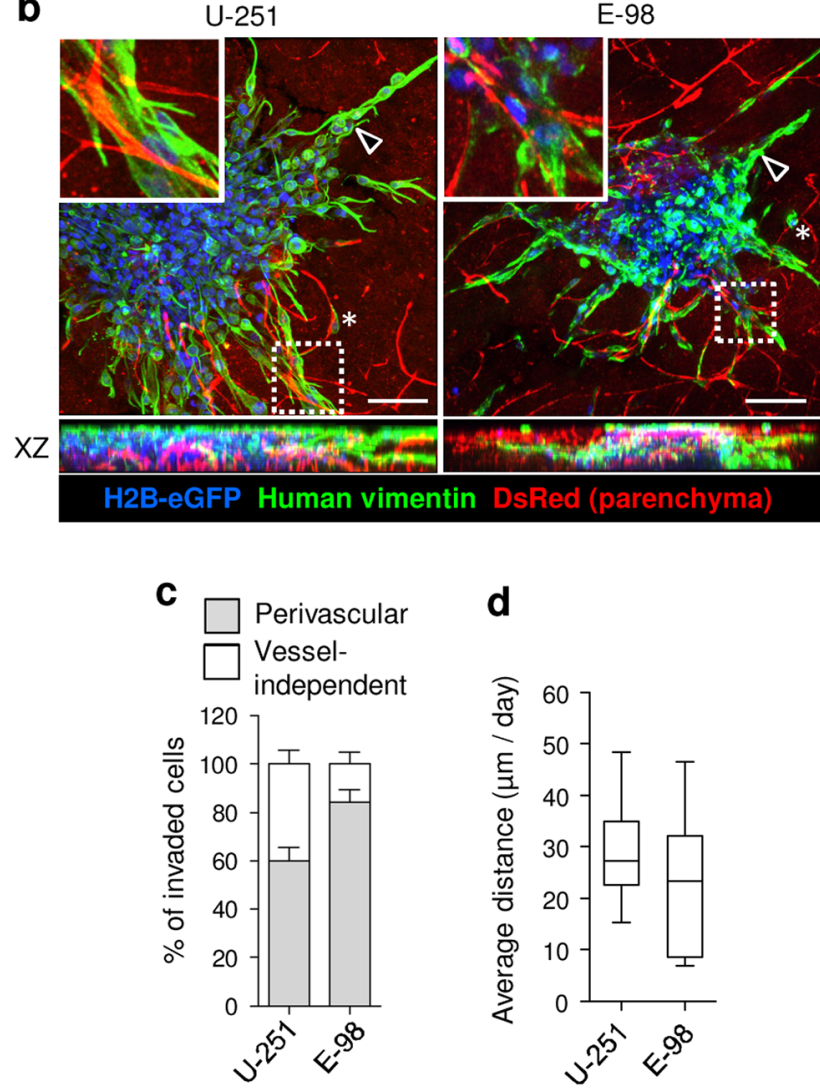

d

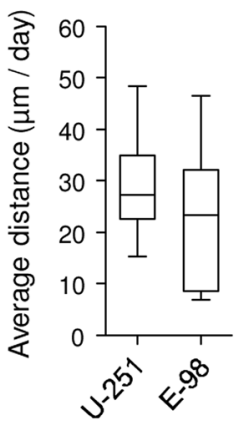

Fig. 4 Organotypic mouse brain slice invasion assay. a Assay design. b $3 \mathrm{D}$ reconstruction (confocal z-stack, $90 \mu \mathrm{m}$, horizontal and orthogonal projections) of U-251 and E-98 cell migration from spheroids in mouse brain slices after 2 days of culture. Arrowheads indicate multicellular strands. Asterisks, detached single cells. Red signal originates from the DsRed mouse background, as contrast of vessels (bright signals) and stromal cells (dim signal). c Fractions of glioma cells associated with blood vessels, identified by vimentin staining with human-specific antibody. d Average distance of U-251 and E-98 cell migration in mouse brain slices. Values display median (black line), 25/75 percentiles (boxes) and maximum/minimum (whiskers) from three independent experiments. Scale bars $100 \mu \mathrm{m}$

equally well supported (Fig. 1b, c). This indicates a generic pro-migratory function of a protein interface adjacent to viscous polysaccharide. In previous work, glioma cell migration was primarily assessed in single-cell migration assays, using cell suspensions after enzymatic dispersion (Nakada et al. 2013; Chen and Nalbantoglu 2014). However, when tested as tumor-like multicellular spheroids, which allow cells to establish cell-cell junctions, both U-251 and E-98

Fig. 5 Validation of in vitro assays by glioma invasion in murine and human brain in vivo. a 3D reconstruction of U-251 cell invasion along rBM-plastic interface in vitro compared to invasion pattern in the mouse brain 1 month after orthotopic implantation of U-251 cells. Glioma cells were identified using human-specific antivimentin, basement membranes with anti-laminin, and astrocytes and glia limitans perivascularis with anti-GFAP antibody. Arrowhead indicates glioma cells invading along basement membrane of a linear brain vessel under glia limitans. V-vessel lumen. In vivo images are projections from $100 \mu \mathrm{m}$-thick z-stacks. b E-468 patient-derived glioblastoma cells invading ( 2 days) 3D astrocyte scaffolds in vitro, or mouse brain 2 months after orthotopic implantation, compared with the peritumoral region of a primary glioblastoma (PGB) patient sample. Images represent $100 \mu \mathrm{m}$-thick z-stacks. Arrowheads denote contacts between glioma cells via dendrite-like filaments. Glioma cells were positive for vimentin (E-468) or nestin (E-468 and human sample), detected with human specific antibodies. Astrocytes were detected by anti-GFAP antibody. c Number of cell-cell junctions between U-251 and E-98 glioma cells in different assays, including collective strands under rBM compared to perivascular invasion in mouse brain. Values represent the number of cell contacts per glioma cell (colour code of stacked boxes) and their relative frequency in the population. The number of connected neighbour cells is indicated as median (red square), 25/75 percentiles (whiskers), representing three independent in vitro experiments and 2 mice per cell line in mouse brain. d Number of filaments connecting glioblastoma cells during astrocyte scaffold invasion compared with mouse brain tissue and primary glioblastoma lesion. Values display median (black line), 25/75 percentiles (boxes) and maximum/minimum (whiskers). Data represent three independent in vitro experiments; two E-468 xenografts in mouse brain, and four glioblastoma patients. Scale bars $50 \mu \mathrm{m}$

cells migrated collectively, as a cohesive sheet of cells, along the rBM-hyaluronan interface (Fig. 1d, e). Similarly, when spheroids were positioned at the interface between $\mathrm{rBM}$ overlaying the plastic substrate of the culture plate (Fig. 2a), U-251 glioma cells migrated as epithelial-like sheets and collective strands, whereas E-98 cells formed thinner strands and complex-shaped multicellular networks with cells retaining both linear junctions and connecting filaments between cell bodies (Fig. 2b, c). Glioma invasion modes observed in hyaluronan-rBM vs rBM-plastic interfaces may result from the different molecular and mechanical characteristics of the interface along which they migrated, including coverage of rBM proteins by hyaluronan which may provide confinement and further modulate ligand availability for cell adhesion systems and the stiffness of migration substrate. These parameters may cooperatively influence the retention of cell-cell junctions, migration mode and speed (Haeger et al. 2014; Canver et al. 2016; Asano et al. 2017; Bangasser et al. 2017). Accordingly, both glioma cell lines moving collectively under rBM established linear or focal adherens junctions at cell-cell contacts which were positive for $\mathrm{N}$-cadherin and $\beta$-catenin, whereas glioma cells migrating on a $2 \mathrm{D}$ surface lacked junctional $\beta$-catenin and showed its redistribution to the cytoplasm (Fig. 2d, e). The average distance of glioma cell migration under rBM was decreased 

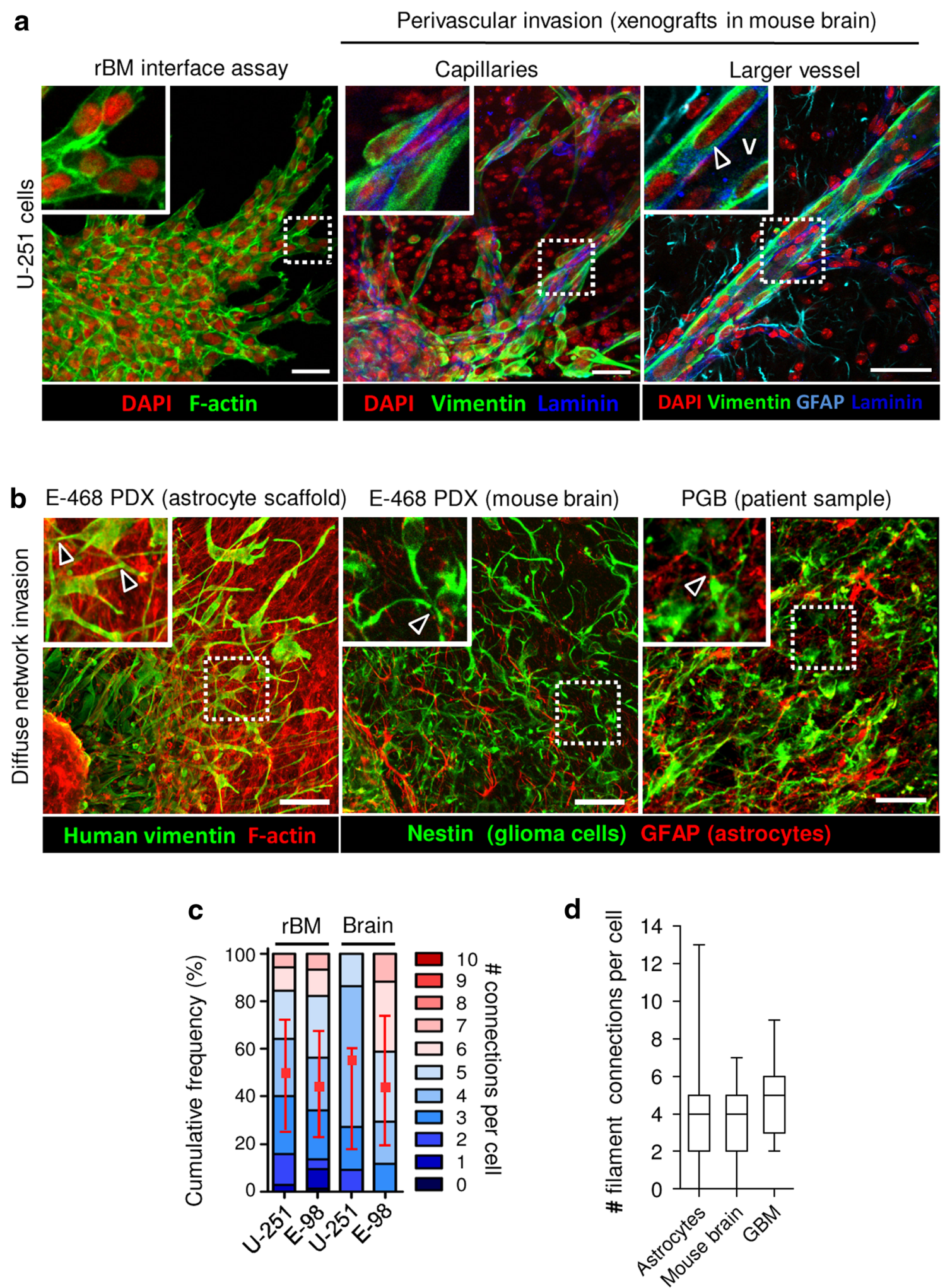
by $60-80 \%$, compared to migration along rBM-hyaluronan interfaces (compare Fig. 2c with Fig. 1c), indicating speed regulation in dependence of variations of confinement and/ or substrate stiffness.

\section{Invasion into 3D astrocyte scaffolds}

To reproduce diffuse glioma cell invasion in astrocyte-rich brain stroma we generated 3D scaffolds formed by immortalized murine astrocytes in hyperconfluent culture (Fig. 3a). Astrocytes proliferated and formed dense multicellular networks with up to three cell layers in thickness $(\sim 35 \mu \mathrm{m})$ during 3 days of culture (Fig. 3b). Astrocytes of the bottom layer typically aligned in parallel, whereas the top layer developed more varied and randomly orientated network-like organization (Fig. 3b). Hyperconfluent astrocyte cultures produced extracellular matrix molecules along their cell boundaries, including laminin and collagen IV (Fig. 3b), resulting in a dense cell- and ECM-rich 3D scaffold.

Glioma cells readily invaded astrocyte scaffolds, by aligning along and intercalating between astrocytes and penetrating all scaffold layers (Fig. 3c). The speed of glioma cell invasion correlated inversely with the duration of astrocyte scaffold conditioning, with average distances covered decreasing from $\sim 100 \mu \mathrm{m} /$ day in 2-day old scaffolds to less than $10 \mu \mathrm{m} /$ day in 10-day old scaffolds (Fig. 3d). Notably, and in contrast to rBM based culture, U-251 and E-98 cells invaded astrocyte scaffolds as both, single cells (Fig. 3c, asterisk) and multicellular networks of individual cells connecting with neighbor cells via long dendrite-like filaments (Fig. 3c, arrowheads).

\section{Invasion into mouse brain slices}

The brain blood vessels have a complex anatomical and molecular organization (Yousif et al. 2013; Di Russo et al. 2017), and in vitro assays fail to reconstitute these microanatomic features. To recapitulate the perivascular niche for U-251 and E-98 cell invasion and validate the results obtained in rBM culture, we used organotypic brain slice culture (Fig. 4a). U-251 and E-98 cells both invaded the brain slice tissue effectively and preferentially associated with blood vessels (Fig. 4b, c). Using end-point analysis of the position of individual cells relative to the spheroid boundary, the invasion speed was $\sim 10-50 \mu \mathrm{m} /$ day (median $\sim 25 \mu \mathrm{m} /$ day) for both cell lines (Fig. 4d), similar to the invasion speed in 3-day matured astrocyte scaffolds (Fig. 3d). The invasion pattern of U-251 and E-98 glioma cells in brain slice culture was adaptive and was dominated by collective cell strands while extending along blood vessels (Fig. 4b, arrowheads) and occasional detached single cells (Fig. 4b, asterisks). Thus, glioma cell invasion from spheroid culture on brain slices displays plastic adaptation in dependence of the tissue subregion.

\section{Validation of in vitro assays by glioma invasion in vivo}

To benchmark each in vitro invasion model, we compared the respective invasion patterns obtained in $\mathrm{rBM}, 3 \mathrm{D}$ astrocyte scaffolds and brain slice cultures with brain invasion in vivo, using 3D reconstructions of patient-derived xenografts in mouse brain and glioblastoma patient samples (Fig. 5a, b). Orthotopically injected in mouse brain, perivascular invasion of U-251 and E-98 glioma cells progressed as collective, finger-like strands along capillaries and larger blood vessels (Fig. 5a), and this pattern was reminiscent to their cohesive strand migration along $\mathrm{rBM}$ interfaces (Fig. 5a). Among other invasion patterns, similar cohesive, strand-like glioma cell invasion along blood vessels were previously observed by intravital two-photon microscopy in the mouse brain (Winkler et al. 2009; Watkins et al. 2014). The number of connections per cell in perivascular invasion strands was similar for in vitro rBM and in vivo mouse models, with $70 \%$ of the cells in direct contact with 3-7 neighbor cells (Fig. 5c). rBM is often used for coating transwell filters to model cell invasion through, rather than along, basement membrane (Benton et al. 2014). However, the data from the perivascular invasion in vivo confirm that glioma cells preferentially migrate along basement membranes and follow the perivascular space, but typically do not intravasate (Farin et al. 2006; Watkins et al. 2014).

Likewise, the in vitro/in vivo correlation was high for multicellular glioma network organization. Glioma cells connected with long filaments were found both in peritumoral regions of E-468 patient derived xenografts (PDX) in the mouse brain and glioblastoma lesions from patient samples (Fig. 5b, arrowheads), which was consistent with the filament-based network-like pattern of E-468 cells (Fig. 5b) during infiltration of 3D astrocyte scaffolds. The number of filament-based connections between E-468 cells in $3 \mathrm{D}$ astrocyte scaffolds (median four connections/cell) matched the number of filamentous connections between E-468 cells in the mouse brain (median four connections/ cell) as well as in human glioblastoma samples (median five connections/cell) (Fig. 5b). The plasticity of glioma cell invasion modes, including cohesive strand- and sheet-like structures along rBM interfaces and in perivascular space, and diffuse multicellular networks in astrocyte scaffolds likely reflect the geometry of the environment, including microtracks of least resistance and confinement effects (Monzo et al. 2016).

Thus, the rBM interface and 3D astrocyte scaffold assays reliably represent two major routes of glioma dissemination in brain tissue in vivo: strand-like collective perivascular 
invasion along basement membranes and multicellular networks in astrocyte-rich stroma. Because rBM interface and astrocyte scaffold invasion assays are performed in 96-well plate format, both assays may be amenable for pharmacological compound screens and other experimental treatments.

Acknowledgements We acknowledge Esther Wagena and Bianca Lemmers-Van de Weem for the excellent support for animal experiments, Anneke Navis for expert technical support for E-468 cell isolation from mouse brain, and Mietske Wijers-Rouw for expert technical assistance in scanning electron microscopy. We are greatful to Amparo Acker-Palmer for providing immortalized mouse astrocytes and Pieter Wesseling for supply of glioblastoma samples. This work was supported by NWO-VICI (918.11.626), Pieken in the Delta Oost Nederland and the Cancer Genomics Center, The Netherlands (to P.F.).

\section{Compliance with ethical standards}

Conflict of interest The authors declare that they have no conflict of interest.

Open Access This article is distributed under the terms of the Creative Commons Attribution 4.0 International License (http:// creativecommons.org/licenses/by/4.0/), which permits unrestricted use, distribution, and reproduction in any medium, provided you give appropriate credit to the original author(s) and the source, provide a link to the Creative Commons license, and indicate if changes were made.

\section{References}

Albini A, Iwamoto Y, Kleinman HK et al (1987) A rapid in vitro assay for quantitating the invasive potential of tumor cells. Cancer Res 47:3239-3245

Alfi STP, Eguerinel CAL, Asset MIM (2002) Invasion of human glioma biopsy specimens in cultures of rodent brain slices: a quantitative analysis. J Neurosurg 97:169-176

Ananthanarayanan B, Kim Y, Kumar S (2011) Elucidating the mechanobiology of malignant brain tumors using a brain matrixmimetic hyaluronic acid hydrogel platform. Biomaterials 32:7913-7923. doi:10.1016/j.biomaterials.2011.07.005

Asano S, Ito S, Takahashi K et al (2017) Matrix stiffness regulates migration of human lung fibroblasts. Physiol Rep 5:e13281. doi:10.14814/phy2.13281

Assanah MC, Bruce JN, Suzuki SO et al (2009) PDGF stimulates the massive expansion of glial progenitors in the neonatal forebrain. Glia 57:1835-1847. doi:10.1002/glia.20895

Baker GJ, Yadav VN, Motsch S et al (2014) Mechanisms of glioma formation: iterative perivascular glioma growth and invasion leads to tumor progression, VEGF-independent vascularization, and resistance to antiangiogenic therapy. Neoplasia 16:543-561. doi:10.1016/j.neo.2014.06.003

Bangasser BL, Shamsan GA, Chan CE et al (2017) Shifting the optimal stiffness for cell migration. Nat Commun 8:15313. doi: $10.1038 /$ ncomms 15313

Bellail AC, Hunter SB, Brat DJ et al (2004) Microregional extracellular matrix heterogeneity in brain modulates glioma cell invasion. Int J Biochem Cell Biol 36:1046-1069. doi:10.1016/j. biocel.2004.01.013
Benton G, Arnaoutova I, George J et al (2014) Matrigel: from discovery and ECM mimicry to assays and models for cancer research. Adv Drug Deliv Rev 79-80:3-18. doi:10.1016/j. addr.2014.06.005

Canver AC, Ngo O, Urbano RL, Clyne AM (2016) Endothelial directed collective migration depends on substrate stiffness via localized myosin contractility and cell-matrix interactions. J Biomech 49:1369-1380. doi:10.1016/j.jbiomech.2015.12.037

Chen H, Nalbantoglu J (2014) Ring cell migration assay identifies distinct effects of extracellular matrix proteins on cancer cell migration. BMC Res Notes 7:183. doi:10.1186/1756-0500-7-183

Claes A, Schuuring J, Boots-sprenger S et al (2008) Phenotypic and genotypic characterization of orthotopic human glioma models and its relevance for the study of anti-glioma therapy. Brain Pathol 18:423-433. doi:10.1111/j.1750-3639.2008.00141.x

Cuddapah VA, Robel S, Watkins S, Sontheimer H (2014) A neurocentric perspective on glioma invasion. Nat Rev Neurosci 15:455465. doi:10.1038/nrn3765

Delpech B, Maingonnat C, Girard N et al (1993) Hyaluronan and hyaluronectin in the extracellular matrix of human brain tumour stroma. Eur J Cancer 29A:1012-1017

Depner C, Zum Buttel H, Bogurcu N et al (2016) EphrinB2 repression through ZEB2 mediates tumour invasion and anti-angiogenic resistance. Nat Commun 7:12329. doi:10.1038/ncomms12329

Di Russo J, Hannocks M-J, Luik A-L et al (2017) Vascular laminins in physiology and pathology. Matrix Biol 57-58:140-148. doi:10.1016/j.matbio.2016.06.008

Farin A, Suzuki SO, Weiker M et al (2006) Transplanted glioma cells migrate and proliferate on host brain vasculature: a dynamic analysis. Glia 53:799-808. doi:10.1002/glia.20334

Fayzullin A, Tuvnes FA, Skjellegrind HK et al (2016) Time-lapse phenotyping of invasive glioma cells ex vivo reveals subtype-specific movement patterns guided by tumor core signaling. Exp Cell Res 349:199-213. doi:10.1016/j.yexcr.2016.08.001

Frolov A, Evans IM, Li N et al (2016) Imatinib and Nilotinib increase glioblastoma cell invasion via Abl-independent stimulation of p130Cas and FAK signalling. Sci Rep 6:27378. doi:10.1038/ srep27378

Gordon VD, Valentine MT, Gardel ML et al (2003) Measuring the mechanical stress induced by an expanding multicellular tumor system: a case study. Exp Cell Res 289:58-66

Gritsenko PG, Ilina O, Friedl P (2012) Interstitial guidance of cancer invasion. J Pathol 226:185-199. doi:10.1002/path.3031

Haeger A, Krause M, Wolf K, Friedl P (2014) Cell jamming: collective invasion of mesenchymal tumor cells imposed by tissue confinement. Biochim Biophys Acta 1840:2386-2395. doi:10.1016/j. bbagen.2014.03.020

Hirata E, Yukinaga H, Kamioka Y et al (2012) In vivo fluorescence resonance energy transfer imaging reveals differential activation of Rho-family GTPases in glioblastoma cell invasion. J Cell Sci 125:858-868. doi:10.1242/jcs.089995

Hong X, Sin WC, Harris AL, Naus CC (2015) Gap junctions modulate glioma invasion by direct transfer of microRNA. Oncotarget 6:15566-15577. doi:10.18632/oncotarget.3904

Hughes CS, Postovit LM, Lajoie GA (2010) Matrigel: a complex protein mixture required for optimal growth of cell culture. Proteomics 10:1886-1890. doi:10.1002/pmic.200900758

Huijbers IJ, Iravani M, Popov S et al (2010) A role for fibrillar collagen deposition and the collagen internalization receptor endo180 in glioma invasion. PLoS One 5:e9808. doi:10.1371/journal. pone. 0009808

Kaufman LJ, Brangwynne CP, Kasza KE et al (2005) Glioma expansion in collagen I matrices: analyzing collagen concentrationdependent growth and motility patterns. Biophys J 89:635-650. doi:10.1529/biophysj.105.061994 
Kitai R, Horita R, Sato K et al (2010) Nestin expression in astrocytic tumors delineates tumor infiltration. Brain Tumor Pathol 27:1721. doi:10.1007/s10014-009-0261-0

Korff T, Augustin HG (1998) Integration of endothelial cells in multicellular spheroids prevents apoptosis and induces differentiation. J Cell Biol 143:1341-1352

Krusche B, Ottone C, Clements MP et al (2016) EphrinB2 drives perivascular invasion and proliferation of glioblastoma stem-like cells. Elife. doi: 10.7554/eLife. 14845

Lenting K, Verhaak R, Ter Laan M et al (2017) Glioma: experimental models and reality. Acta Neuropathol 133:263-282. doi:10.1007/ s00401-017-1671-4

Miao H, Gale NW, Guo H et al (2015) EphA2 promotes infiltrative invasion of glioma stem cells in vivo through cross-talk with Akt and regulates stem cell properties. Oncogene 34:558-567. doi: $10.1038 /$ onc. 2013.590

Mir SE, De Witt Hamer PC, Krawczyk PM et al (2010) In silico analysis of kinase expression identifies WEE1 as a gatekeeper against mitotic catastrophe in glioblastoma. Cancer Cell 18:244-257. doi:10.1016/j.ccr.2010.08.011

Montana V, Sontheimer H (2011) Bradykinin promotes the chemotactic invasion of primary brain tumors. J Neurosci 31:4858-4867. doi:10.1523/JNEUROSCI.3825-10.2011

Monzo P, Chong YK, Guetta-Terrier C et al (2016) Mechanical confinement triggers glioma linear migration dependent on formin FHOD3. Mol Biol Cell 27:1246-1261. doi:10.1091/mbc. E15-08-0565

Motegi H, Kamoshima Y, Terasaka S et al (2014) Type 1 collagen as a potential niche component for CD133-positive glioblastoma cells. Neuropathology 34:378-385. doi:10.1111/neup.12117

Nakada M, Nambu E, Furuyama N et al (2013) Integrin $\alpha 3$ is overexpressed in glioma stem-like cells and promotes invasion. $\mathrm{Br} \mathrm{J}$ Cancer 108:2516-2524. doi:10.1038/bjc.2013.218

Oliveira R, Christov C, Guillamo JS et al (2005) Contribution of gap junctional communication between tumor cells and astroglia to the invasion of the brain parenchyma by human glioblastomas. BMC Cell Biol 6:1-17. doi:10.1186/1471-2121-6-7

Osswald M, Jung E, Sahm F et al (2015) Brain tumour cells interconnect to a functional and resistant network. Nature 528:93-98. doi:10.1038/nature16071

Osswald M, Solecki G, Wick W, Winkler F (2016) A malignant cellular network in gliomas: potential clinical implications. Neuro Oncol 18:479-485. doi:10.1093/neuonc/now014

Payne LS, Huang PH (2013) The pathobiology of collagens in glioma. Mol Cancer Res 11:1129-1140. doi:10.1158/1541-7786. MCR-13-0236

Rao SS, Lannutti JJ, Viapiano MS et al (2014) Toward 3D biomimetic models to understand the behavior of glioblastoma multiforme cells. Tissue Eng Part B Rev 20:314-327. doi:10.1089/ten. TEB.2013.0227

Rape A, Ananthanarayanan B, Kumar S (2014) Engineering strategies to mimic the glioblastoma microenvironment. Adv Drug Deliv Rev 79-80:172-183. doi:10.1016/j.addr.2014.08.012

Rath BH, Fair JM, Jamal M et al (2013) Astrocytes enhance the invasion potential of glioblastoma stem-like cells. PLoS One 8:e54752. doi:10.1371/journal.pone.0054752

Sawamiphak S, Seidel S, Essmann CL et al (2010) Ephrin-B2 regulates VEGFR2 function in developmental and tumour angiogenesis. Nature 465:487-491. doi:10.1038/nature08995

Schindelin J, Arganda-Carreras I, Frise E et al (2012) Fiji: an opensource platform for biological-image analysis. Nat Methods 9:676-682. doi:10.1038/nmeth.2019

Sykova E, Nicholson C (2008) Diffusion in brain extracellular space. Physiol Rev 88:1277-1340. doi:10.1152/physrev.00027.2007

Ulrich TA, de Juan Pardo EM, Kumar S (2009) The mechanical rigidity of the extracellular matrix regulates the structure, motility, and proliferation of glioma cells. Cancer Res 69:4167-4174. doi:10.1158/0008-5472.CAN-08-4859

Watkins S, Robel S, Kimbrough IF et al (2014) Disruption of astrocytevascular coupling and the blood-brain barrier by invading glioma cells. Nat Commun 5:4196. doi:10.1038/ncomms5196

Wen PY, Reardon DA (2016) Neuro-oncology in 2015: progress in glioma diagnosis, classification and treatment. Nat Rev Neurol 12:69-70. doi:10.1038/nrneurol.2015.242

Winkler F, Kienast Y, Fuhrmann M et al (2009) Imaging glioma cell invasion in vivo reveals mechanisms of dissemination and peritumoral angiogenesis. Glia 57:1306-1315. doi:10.1002/glia.20850

Wurdinger T, Badr C, Pike L et al (2008) A secreted luciferase for ex vivo monitoring of in vivo processes. Nat Methods 5:171-173. doi:10.1038/nmeth.1177

Yang Y, Motte S, Kaufman LJ (2010) Pore size variable type I collagen gels and their interaction with glioma cells. Biomaterials 31:5678-5688. doi:10.1016/j.biomaterials.2010.03.039

Yousif LF, Di Russo J, Sorokin L (2013) Laminin isoforms in endothelial and perivascular basement membranes. Cell Adhes Migr 7:101-110. doi:10.4161/cam.22680

Zimmermann DR, Dours-Zimmermann MT (2008) Extracellular matrix of the central nervous system: from neglect to challenge. Histochem Cell Biol 130:635-653. doi:10.1007/s00418-008-0485-9 